\title{
Sustained improvement in staff influenza vaccination rates over six years without a mandatory policy
}

\author{
Caroline Marshall MBBS, FRACP, PhD, Grad Dip Clin Epi ${ }^{1,2,3}$ (1), Kathryn Williams RN ${ }^{1}$, \\ Elizabeth Matchett RN, Grad Dip Ad Nursing ${ }^{1}$ and Louise Hobbs RN, PhD ${ }^{1}$ \\ ${ }^{1}$ Infection Prevention and Surveillance Service, Royal Melbourne Hospital, Melbourne, Victoria, Australia, ${ }^{2}$ Victorian Infectious Diseases Service, Peter Doherty \\ Institute of Infection and Immunity, Melbourne, Victoria, Australia and ${ }^{3}$ Department of Medicine, University of Melbourne, Melbourne, Victoria, Australia
}

To the Editor - Annual healthcare worker (HCW) influenza vaccination is recommended, yet compliance is often poor. In 2012, the rate at our institution was $43 \%$. We report sustained improvement over 6 years without a mandate. Our free-of-charge vaccination program covers $>8,000$ staff across 32 campuses: a tertiary-care referral hospital, a subacute rehabilitation and aged care campus, and multiple smaller sites including dialysis, mental health, and residential long-term care facilities.

\section{Program components}

1. Executive support, strong leadership, and governance were provided. A multidisciplinary subcommittee met fortnightly and reported to the Infection Prevention Committee. In 2013, a Key Performance Indicator of 95\% for declaration forms was set. The declaration required each HCW either to be vaccinated, to indicate vaccination elsewhere, or to decline vaccine.

2. Prior to 2013, \$24 USD (\$33 AUD) per employee was charged to the organizational divisions according to the number vaccinated. From 2013, charges were applied according to how many were employed, regardless of vaccination uptake.

3. Staff influenza vaccination rates are included in the Victorian state government health service performance monitoring framework, with a target of $75 \%$, which increased to $80 \%$ in 2018.

4. A full-time influenza vaccination coordinator was appointed for 6 months each year. Nurse immunizers covered all shifts daily for 8 weeks in 2011, 9 weeks in 2012, 10 weeks in 2013, 8 weeks in 2014, and 6 weeks each year from 2015 to 2018.

5. From 2006 onward, vaccinators attended all campuses at least once. A "Flu Stop Shop" was set up from 2013 onward in the main campus foyer, providing vaccinators at all times for walk-ins during the campaign. Free vaccination was also available without appointment at the staff health clinic throughout the season.

6. A public relations campaign included a dedicated intranet page with links to educational sites, posters in public areas, and floor and elevator stickers. The chief executive officer sent e-mails and gave presentations. Vaccinated staff were given

Author for correspondence: Caroline Marshall, E-mail: caroline.marshall@mh.org.au PREVIOUS PRESENTATION: These data were presented in part at the Australian College for Infection and Prevention Annual Conference, November 2016 in Melbourne, Australia.

Cite this article: Marshall C, et al. (2019). Sustained improvement in staff influenza vaccination rates over six years without a mandatory policy. Infection Control \& Hospital Epidemiology, 40: 389-390, https://doi.org/10.1017/ice.2018.365 stickers or badges and a candy treat. Prizes were given to wards that achieved target levels.

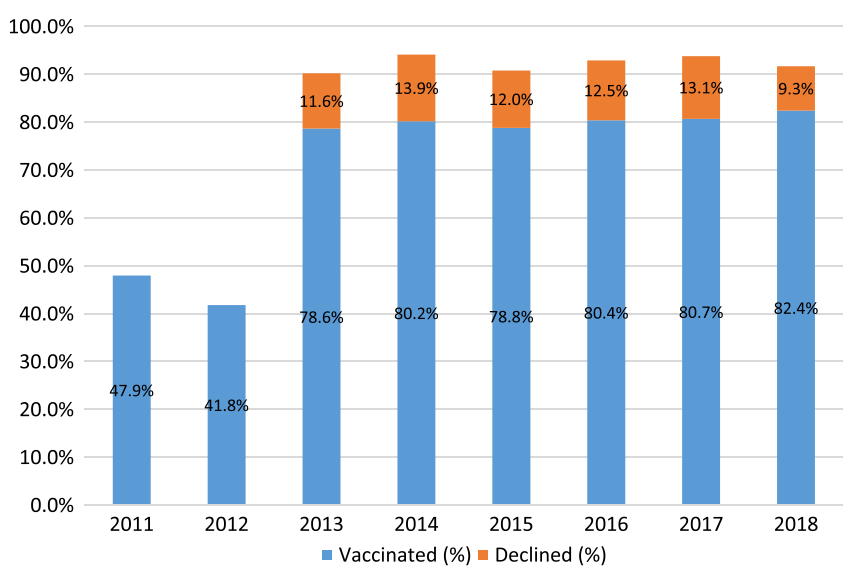

Fig. 1. Proportion of staff that received influenza vaccination or declined vaccination. The proportions that declined in 2011 and 2012 were not available. Refining the denominator from 2013 excluded $\sim 1,000$ staff not working during the influenza season, giving a percentage vaccinated of $\sim 54 \%$ in 2011 and $47 \%$ in 2012 .

7. From 2013, all staff were expected to fill out a "declaration form", comprising the consent form for staff agreeing to be vaccinated and a declination section for staff who did not or who had been vaccinated elsewhere

8. A database was developed, and real-time reports were available via the hospital intranet. Managers were expected to access these reports and to follow-up with the noncompliant staff. Short message service (SMS) messages were sent to noncompliant staff in 2018.

Figure 1 shows the proportion of staff vaccinated. Total compliance with the declaration form was $90.2 \%$ in $2013,94.1 \%$ in $2014,90.8 \%$ in $2015,92.9 \%$ in $2016,93.8 \%$ in 2017 and $91.7 \%$ in 2018. The number vaccinated among the total staff was 3,824 of 7,975 in 2011, 3,675 of 8,794 in 2012, 5,997 of 7,625 in 2013, 6,090 of 7,596 in $2014,5,921$ of 7,512 in $2015,6,334$ of 7,880 in 2016, 6,371 of 7,894 in 2017, and 7,372 of 8,944 in 2018 .

Overall, the campaign cost $\$ 61,275$ USD (\$84,282 AUD) in 2012, $\$ 141,120$ USD (\$194,711 AUD) in 2013, \$126,109 USD $(\$ 174,000$ AUD) in $2014, \$ 149,040$ USD $(\$ 205,640$ AUD) in $2015, \$ 139,930$ USD (\$193,070 AUD) in 2016, $\$ 167,289$ USD (\$230,819 AUD) in 2017, and \$136,032 USD (\$187,692 AUD) in 2018. Costs consisted largely of employing vaccinators and to 
a lesser extent, obtaining "Flu Stop Shop" space, equipment, advertising and educational materials, and prizes and gifts.

We have demonstrated that a sustained improvement in HCW influenza vaccination and declaration rates is possible without a mandatory policy. Although we had a moderate declination rate, overall we engaged $>90 \%$ of staff who either had the vaccine or declined.

Unwavering hospital board and executive support, a strong communication strategy, and dedicated funding were crucial. Devolvement of responsibility to managers in ensuring that staff were vaccinated was facilitated by real-time feedback of vaccination rates.

Monitoring compliance with the vaccination program (vaccination rates plus signed declaration forms) as a whole is suggested as a metric as well as monitoring actual vaccination rates where mandatory vaccination is not in place. ${ }^{1}$ Anecdotally, some staff were vaccinated elsewhere without informing us. To ensure higher rates, we would have to make vaccination mandatory, a step we are reluctant to take, given that there is no such directive in our state and because we have achieved very good staff engagement without.

A study of Australian stakeholders demonstrated strong support for voluntary but not mandatory HCW influenza vaccination. $^{2}$ Another found that only $51 \%$ favored mandatory vaccination. ${ }^{3}$ Anecdotal evidence suggests that our staff would not favor a mandatory policy. Some refused to sign the declaration form, believing this was an encroachment on their autonomy. This finding is similar to another Melbourne hospital, which reported that $5.4 \%$ of unvaccinated staff had not completed a declaration form because they "did not wish to."

In our opinion, the case for mandatory vaccination does not outweigh the ethical arguments against. Vaccination is neither necessary nor sufficient to prevent influenza transmission in the healthcare setting. ${ }^{5}$ Many US professional bodies endorse mandatory influenza vaccine, while others oppose it. Vaccination rates of nearly $100 \%$ have been achieved in organizations with mandatory policies, with termination of staff who refuse. ${ }^{6,7}$ Others have achieved rates $>90 \%$ without a mandate, although some used other incentives including financial rewards. ${ }^{8,9}$

We demonstrated sustained improvement in staff influenza vaccination rates without a mandate or incentives. We do not believe a mandatory policy would be well accepted by our staff, and given that it will not prevent all healthcare-associated influenza, this is not something we plan to introduce. Our program was successful and sustainable, but it was also cost and labor intensive.

Author ORCIDs. Caroline Marshall, (D) 0000-0001-5901-2004

Acknowledgments. We thank Barry Rosenberg and Chris Ott for providing data.

Financial support. No financial support was provided relevant to this article.

Conflicts of interest. C.M. reports that her spouse holds a small number of shares in CSL, which makes an influenza vaccine. All other authors report no conflicts of interest relevant to this article.

\section{References}

1. Wang D, Worth L, Bull A, Bennett N, Richards M. Influenza vaccination of Victorian healthcare workers: will a higher target increase vaccine uptake? Aust NZ J Public Health 2014;38:490.

2. Lim YC, Seale H. Examining the views of key stakeholders regarding the provision of occupational influenza vaccination for healthcare workers in Australia. Vaccine 2014;32:606-610.

3. Leask J, Helms CM, Chow MY, Robbins SC, McIntyre PB. Making influenza vaccination mandatory for health care workers: the views of NSW Health administrators and clinical leaders. NSW Public Health Bull 2010;21: 243-247.

4. Heinrich-Morrison K, McLellan S, McGinnes U, et al. An effective strategy for influenza vaccination of healthcare workers in Australia: experience at a large health service without a mandatory policy. BMC Infect Dis 2015;15:42.

5. Cheng AC, Worth LJ. Mandatory influenza vaccination of healthcare workers: is it necessary or sufficient to protect patients? Healthcare Infect 2014; 19:114-115.

6. Babcock HM, Gemeinhart N, Jones M, Dunagan WC, Woeltje KF. Mandatory influenza vaccination of health care workers: translating policy to practice. Clin Infect Dis 2010;50:459-464.

7. Rakita RM, Hagar BA, Crome P, Lammert JK, Mandatory influenza vaccination of healthcare workers: a 5-year study. Infect Control Hosp Epidemiol 2010;31:881-888.

8. Drees M, Wroten K, Smedley M, Mase T, Schwartz JS. Carrots and sticks: achieving high healthcare personnel influenza vaccination rates without a mandate. Infect Control Hosp Epidemiol 2015;36:717-724.

9. Honda H, Sato Y, Yamazaki A, Padival S, Kumagai A, Babcock H. A successful strategy for increasing the influenza vaccination rate of healthcare workers without a mandatory policy outside of the United States: a multifaceted intervention in a Japanese tertiary care center. Infect Control Hosp Epidemiol 2013;34:1194-1200. 